\title{
Light Scattering, Absorption and Refraction due to High-Order Optical Nonlinearities in Colloidal Gold Nanorods (Supporting Information)
}

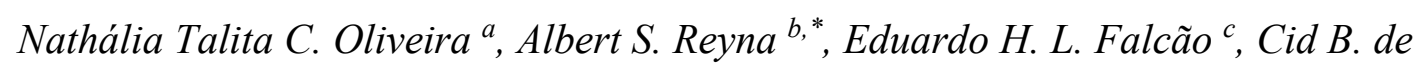
Araújo $^{a, d}$

a Programa de Pós-Graduação em Ciência de Materiais, Universidade Federal de Pernambuco, Recife, PE 50740-560, Brazil.

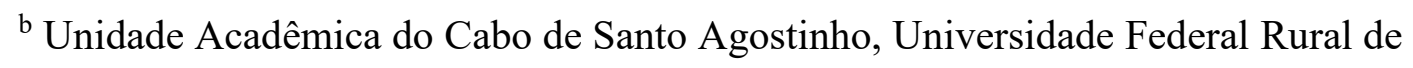
Pernambuco, Cabo de Santo Agostinho, PE 54518-430, Brazil.

c Departamento de Química Fundamental, Universidade Federal de Pernambuco, Recife, PE 50740-560, Brazil.

d Departamento de Física, Universidade Federal de Pernambuco, Recife, PE 50670-901, Brazil.

* Corresponding author. E-mail: areynao@yahoo.com.br 


\section{The intensity-dependent extinction coefficient for gold nanorods}

Figure S1 shows a comparison between four expressions that present the nonlinear (NL) extinction coefficient of gold nanorods as a function of the laser intensity, that were fitted to the experimental results of the open-aperture Z-scan technique, for sample A. The green lines correspond to a standard NL model for metal anisotropic nanoparticles (see Ref. [37]), with excitation frequency close to the localized surface plasmon resonance, which considers the one-photon saturable absorption and intensity-independent two-photon absorption coefficient, i.e. $\alpha(I)=\frac{\alpha_{0}}{1+\sqrt{I / I_{s}}}+\alpha_{2} I$. To apply this expression $\alpha_{0}$ was extracted from the absorption spectrum of the sample, as indicated in Table 1 , and both $\alpha_{2}$ and $I_{s}$ are free parameters for fitting. This model typically describes the change from saturable absorption (SA) to reverse saturable absorption (RSA), a behavior contrary to what was observed in our experiments. Although a valley in transmittance curve for $\mathrm{z}=0$ (focus position) can be observed for low intensities when $I_{S}$ is large, the expression fails to model the saturation effects at high intensities.

As the experimental results show that in low intensities the effects of NL extinction are dominant, it may seems reasonable that $\alpha(I)$ should be expressed by $\alpha_{0}+\alpha_{2} I$. However, the contribution of $\alpha_{2}$ would cause a progressive increase of the valley in the transmittance curve, behavior that is not observed in the present Z-scan experiments, which show a limit in the curve dip. Thus, the intensity-dependent extinction coefficient has to be expressed as $\alpha(I)=\alpha_{0}+\frac{\alpha_{2} I}{1+I / I_{\alpha}}$, where $I_{\alpha}$ represents the saturation intensity of the third-order extinction effect. Such a model is represented in fig. S1 by the blue lines. However, we observed that for intensities larger than $1.6 \mathrm{GW} / \mathrm{cm}^{2}$, the model does not explain the inversion in the transmittance profile for the sample positions around the focal plane.

A model that considers the change from RSA-like to SA-like process, through a $\mathrm{N}-t h$ order process, can be written as: $\alpha(I)=\alpha_{0}+\frac{\alpha_{2} I}{1+I / I_{\alpha}}+\eta I^{(N-1) / 2}$, where $\eta<0$. The black lines illustrate the Z-scan transmittance curves, for $N=3$, which is suitably described up to intensities of $4 \mathrm{GW} / \mathrm{cm}^{2}$. However, an abrupt growth at the transmittance peak is clearly observed for high intensities. Models with $N=5$ or higher values diverge from the experimental results even below $4 \mathrm{GW} / \mathrm{cm}^{2}$, since the increase in transmittance around the focal plane is larger. 
Finally, the red lines correspond to the model adopted in our work, given by equation 1, and previously used by Ref. [53], which models the behavior of the extinction coefficient of gold nanorods, excited in $532 \mathrm{~nm}$, for all intensities used in the experiments.
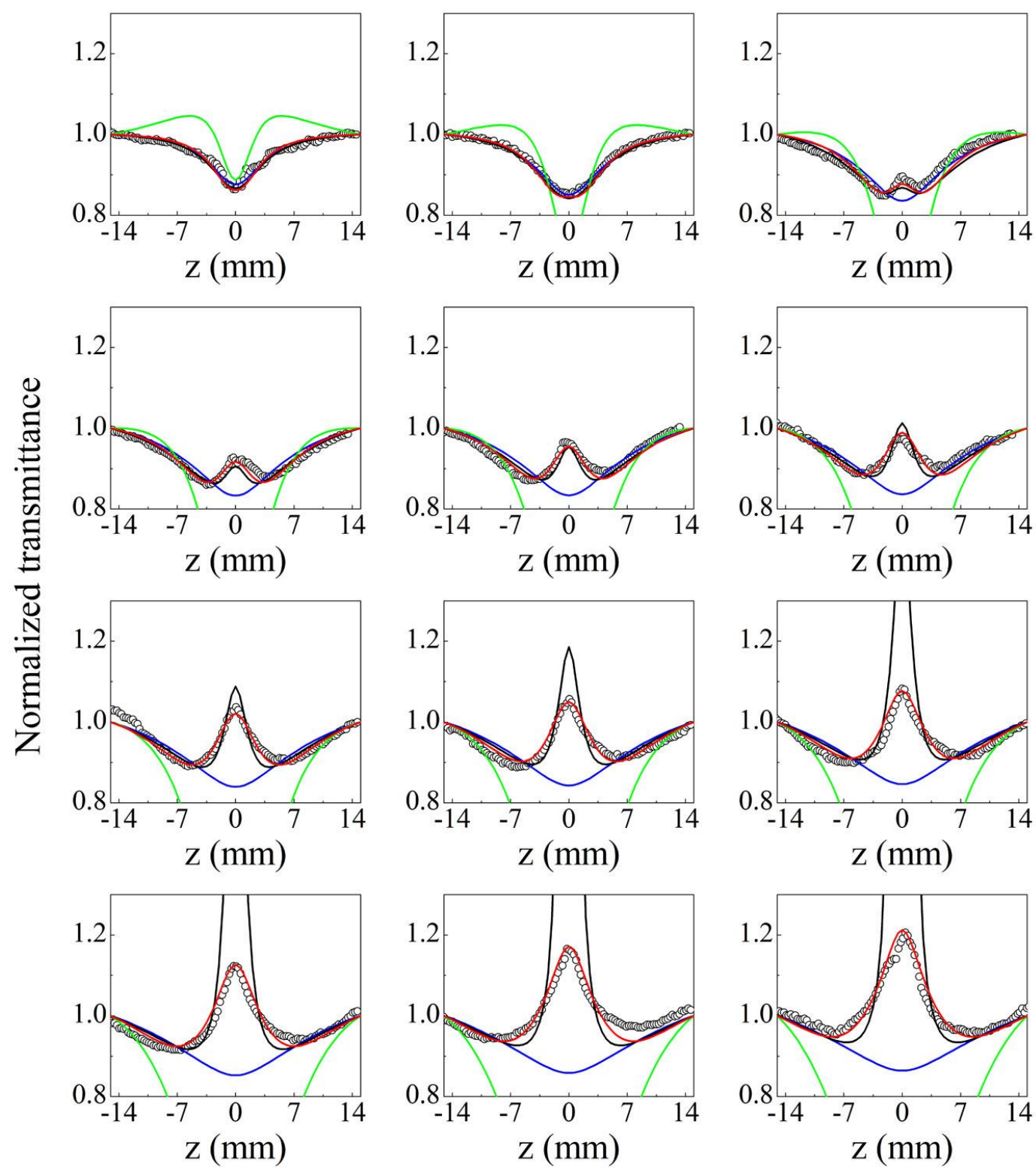

FIG. S1: Experimental (circles) and numerical (lines) open-aperture Z-scan curves, for sample A, with intensities of $0.4,0.8,1.6,2.4,3.2,4.0,4.7,5.5,6.3,7.9,9.5$ and $11.1 \mathrm{GW} / \mathrm{cm}^{2}$. The different models used for the numerical fits are described in section 1 of the supplementary material. 


\section{Open-aperture $\mathrm{Z}$-scan measurements for sample $\mathrm{B}$ and $\mathrm{C}$ with nanorods having different aspect ratios}

According to figure 5, analogous experiments varying the volume fraction and the incident intensity were carried out for samples B and C, with nanorods having aspect ratios of 2.8 and 1.8, respectively. In figs. S2 and S3, the blue circles represent the experimental results measured using the open-aperture Z-scan technique, while the solid lines illustrate the best fit obtained by using the NL extinction coefficient given by $\alpha(I)=\alpha_{0}+\alpha^{N L}(I)+\gamma I^{2}$, as described in the manuscript.
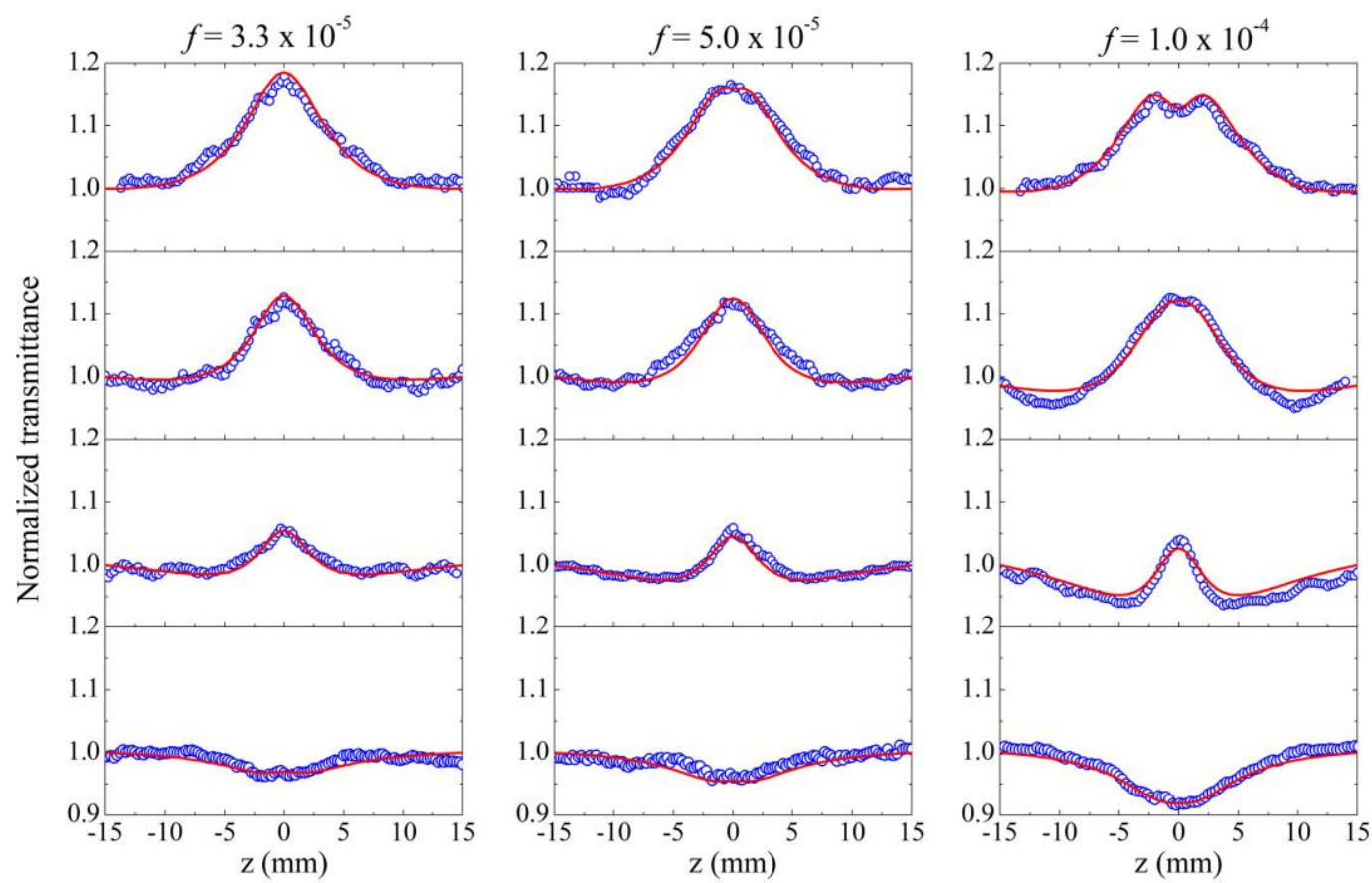

FIG. S2: Open-aperture Z-scan curves for sample B (AR $=2.8)$, with volume fractions of $f=3.3 \mathrm{x}$ $10^{-5}, 5.0 \times 10^{-5}$ and $1 \times 10^{-4}$ and intensities of $0.4,2.4,5.5$ and $11.1 \mathrm{GW} / \mathrm{cm}^{2}$ (from bottom to top). 

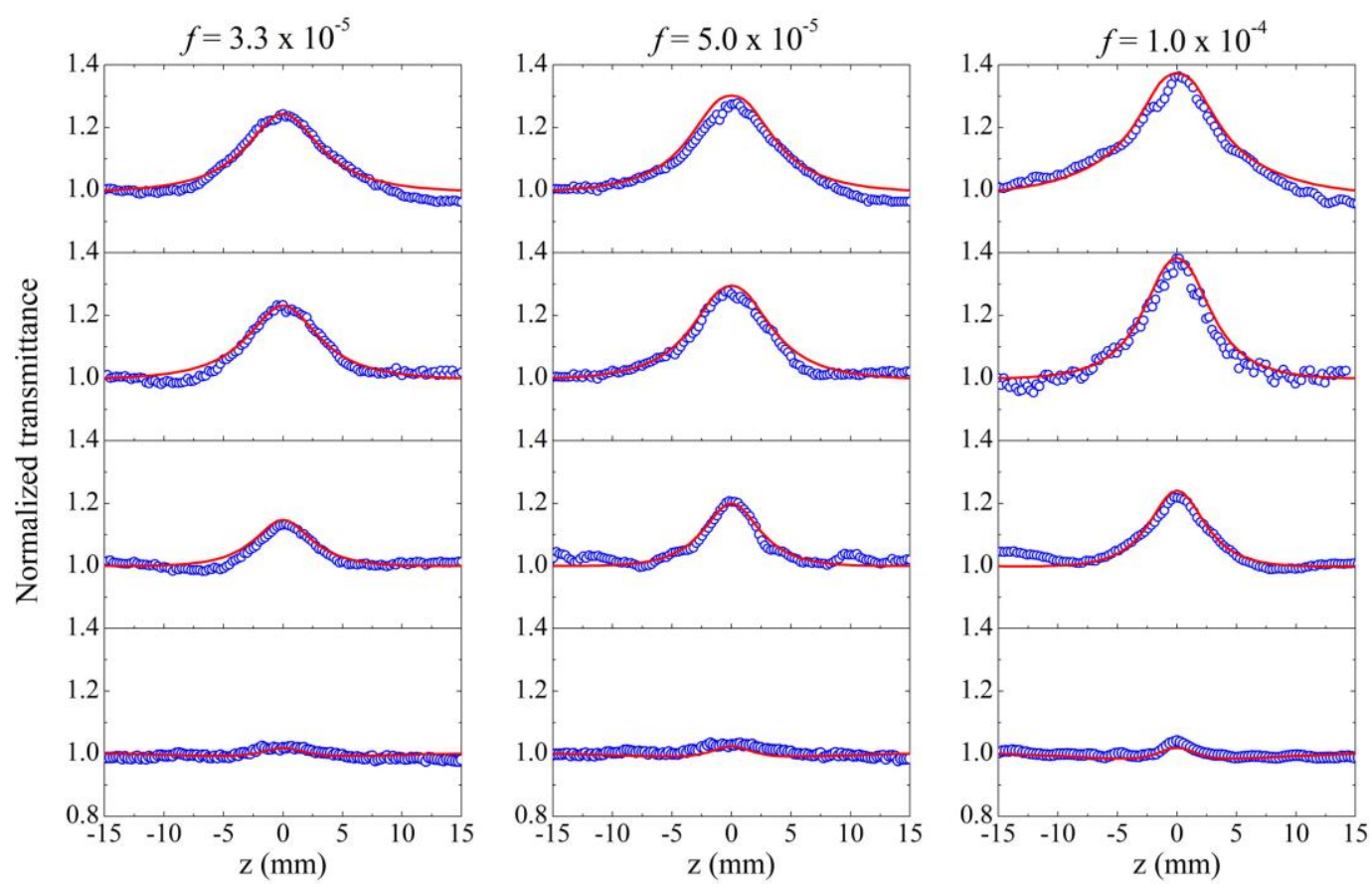

FIG. S3: Open-aperture Z-scan curves for sample C $(\mathrm{AR}=1.8)$, with volume fractions of $f=3.3 \mathrm{x}$ $10^{-5}, 5.0 \times 10^{-5}$ and $1 \times 10^{-4}$ and intensities of $0.4,2.4,5.5$ and $6.3 \mathrm{GW} / \mathrm{cm}^{2}$ (from bottom to top).

The good agreement between the experimental and numerical results for the samples containing gold nanorods with variable sizes support the NL extinction model proposed in the manuscript.

\section{Generalized Maxwell-Garnett model, considering quintic nonlinearities, for metal nanoellipsoids}

The effective cubic and quintic susceptibilities, expressed in Eqs. (3) and (4), can be obtained from the generalized Maxwell-Garnett model, by assuming a diluted homogeneous medium with small filling fraction $(f \ll 1)$ consisting of metal nanoellipsoids suspended in a dielectric medium. Initially, we consider an optical field, $\boldsymbol{E}$, which is uniform on each particle. In the quasi-static approximation, the optical polarization can be written as:

$$
\boldsymbol{P}=\boldsymbol{P}_{h}+\frac{1}{V} \sum_{j=1}^{N_{N P}} \boldsymbol{p}_{j}
$$

where $\boldsymbol{P}_{h}$ is associated to the host medium, $N_{N P}$ is the number of nanoparticles inside the volume $\mathrm{V}$ and $\boldsymbol{p}_{j}$ is the induced dipole moment of each nanoellipsoid. Since the dipole moment of each nanoellipsoid depends on its relative orientation with the applied field, it is necessary to perform an orientational average that 
calculates the effective properties of a composite formed of randomly oriented nanoellipsoids. Then, we have that the second term of the eq. (S.1) can be expressed by $\frac{N_{N P}}{V}\left\langle\boldsymbol{p}_{j}\right\rangle=\varepsilon_{h} \frac{N_{N P}}{V} v_{j}\langle\overleftrightarrow{\sigma} \boldsymbol{E}\rangle$, with $\varepsilon_{h}$ being the dielectric function of the host, $v_{j}$ is the nanoellipsoid volume, $\overleftrightarrow{\sigma}$ is the polarizability density tensor, and $f=\frac{N_{N P}}{V} v_{j}$ is the volume fraction. In terms of the Euler angles $(\theta, \phi, \psi)$, it is possible to represent $\langle\overleftrightarrow{\sigma} \boldsymbol{E}\rangle$ as:

$$
\langle\vec{\sigma} \boldsymbol{E}\rangle=\frac{\int_{0}^{\pi} \int_{0}^{2 \pi} \int_{0}^{2 \pi}\left(\vec{A}^{-1} \vec{\alpha} \vec{A}\right) \sin \theta \mathrm{d} \theta \mathrm{d} \phi \mathrm{d} \psi}{\int_{0}^{\pi} \int_{0}^{2 \pi} \int_{0}^{2 \pi} \sin \theta \mathrm{d} \theta \mathrm{d} \phi \mathrm{d} \psi} \boldsymbol{E}=\sum_{j=1}^{3} \frac{\sigma_{j}}{3} \boldsymbol{E},
$$

where $\overleftrightarrow{A}$ is the rotation matrix. Hence, the electric displacement field in the composite can be expressed as:

$$
\boldsymbol{D}=\varepsilon_{0} \varepsilon_{h}\left(1+f \sum_{j=1}^{3} \frac{\sigma_{j}}{3}\right) \boldsymbol{E},
$$

which depends on the dielectric function of the nanoparticle through $\sigma_{j}=\sigma_{j}\left(\varepsilon_{N P}\right)$. By considering the third- and fifth-order susceptibilities of the metal nanoparticles, we can express $\varepsilon_{N P}$ by:

$$
\varepsilon_{N P}=\varepsilon_{N P}^{L}+\varepsilon_{N P}^{N L} \approx \varepsilon_{N P}^{L}+\frac{3}{4} \chi_{N P}^{(3)}\left\langle\left|\boldsymbol{E}_{N P}\right|^{2}\right\rangle+\frac{5}{8} \chi_{N P}^{(5)}\left\langle\left|\boldsymbol{E}_{N P}\right|^{4}\right\rangle .
$$

To obtain the effective nonlinear susceptibilities, first we must expand $\sigma_{j}$ as a Taylor series in $x=\sqrt{\left\langle\left|E_{N P}\right|^{2}\right\rangle}$. Then, by substituting in eq. (S.3) we have:

$$
\boldsymbol{D} \approx \varepsilon_{0} \varepsilon_{h}\left\{1+\frac{f}{3} \sum_{j=1}^{3}\left[\sigma_{j}\left(\varepsilon_{N P}^{L}\right)+\left.\frac{1}{2 !} \frac{\partial^{2} \sigma_{j}\left(\varepsilon_{N P}\right)}{\partial x^{2}}\right|_{x=0}\left\langle\left|\boldsymbol{E}_{N P}\right|^{2}\right\rangle+\left.\frac{1}{4 !} \frac{\partial^{4} \sigma_{j}\left(\varepsilon_{N P}\right)}{\partial x^{4}}\right|_{x=0}\left\langle\left|\boldsymbol{E}_{N P}\right|^{4}\right\rangle\right]\right\} \boldsymbol{E} .
$$

The electric field $\boldsymbol{E}_{N P}$ can be expressed in terms of the applied electric field, $\boldsymbol{E}$, polarized in the $j$-direction of the ellipsoid as $\left\langle\left|\boldsymbol{E}_{N P}\right|^{2}\right\rangle=\left|\beta_{j}\left(\varepsilon_{\mathrm{NP}}\right)\right|^{2}|\boldsymbol{E}|^{2}$, with:

$$
\beta_{j}\left(\varepsilon_{N P}\right)=\frac{\varepsilon_{h}}{\varepsilon_{h}+\left(\varepsilon_{N P}-\varepsilon_{h}\right) P_{j}},
$$

where $P_{j}$ is the depolarization factor, as reported in [Link, S. et al., J. Phys. Chem. B 1999, 103, 3073-3077]. However, assuming that the ellipsoids are randomly ordered, a second expansion should be performed for $\left|\beta_{j}\left(\varepsilon_{\mathrm{NP}}\right)\right|^{2}$ as function of $x$ that assumes the form 


$$
\begin{aligned}
\left\langle\left|\boldsymbol{E}_{N P}\right|^{2}\right\rangle & =\frac{1}{3} \sum_{j=1}^{3}\left|\beta_{j}\left(\varepsilon_{N P}\right)\right|^{2}|\boldsymbol{E}|^{2} \\
& =\frac{1}{3} \sum_{j=1}^{3}\left|\beta_{j}\left(\varepsilon_{N P}^{L}\right)\right|^{2}|\boldsymbol{E}|^{2}\left\{1+\left.\frac{1}{6} \sum_{k=1}^{3} \frac{\partial^{2}\left|\beta_{k}\left(\varepsilon_{N P}\right)\right|^{2}}{\partial x^{2}}\right|_{x=0}|\boldsymbol{E}|^{2}\right\} .
\end{aligned}
$$

Then, by substituting Eq. (S.7) in Eq. (S.5) and comparing with the electric displacement field, written in the form:

$$
\boldsymbol{D}=\varepsilon_{0}\left[\varepsilon_{\text {eff }}^{L}+\frac{3}{4} \chi_{\text {eff }}^{(3)}\left\langle|\boldsymbol{E}|^{2}\right\rangle+\frac{5}{8} \chi_{\text {eff }}^{(5)}\left\langle|\boldsymbol{E}|^{4}\right\rangle\right] \boldsymbol{E},
$$

it is possible to obtain the effective third- and fifth-order susceptibilities as:

$$
\begin{gathered}
\chi_{\text {eff }}^{(3)}=\varepsilon_{h} f \sum_{j, k=1}^{3}\left[\frac{2}{9} \frac{\partial^{2} \sigma_{j}\left(\varepsilon_{N P}\right)}{\partial x^{2}} \frac{\left|\beta_{k}\left(\varepsilon_{N P}\right)\right|^{2}}{3}\right]_{x=0}, \\
\chi_{\text {eff }}^{(5)}=f \sum_{j=1}^{3}\left[\frac{8 \varepsilon_{h}}{3(5 !)} \frac{\partial^{4} \sigma_{j}\left(\varepsilon_{N P}\right)}{\partial x^{4}}\left(\sum_{k=1}^{3} \frac{\left|\beta_{k}\left(\varepsilon_{N P}\right)\right|^{2}}{3}\right)^{2}\right]_{x=0}+\left.\frac{1}{5} \chi_{\text {eff }}^{(3)} \sum_{j=1}^{3} \frac{\partial^{2}\left(\left|\beta_{j}\left(\varepsilon_{N P}\right)\right|^{2}\right)}{\partial x^{2}}\right|_{x=0},
\end{gathered}
$$

where the NL contribution of the host medium was neglected considering water as the host medium. Then, by solving the Laplace equation, it can be found the polarizability of an ellipsoidal inclusion, given by [Markel, V. A., J. Opt. Soc. Am. A 2016, 33, 1244-1256]:

$$
\sigma_{j}=\frac{\varepsilon_{N P}-\varepsilon_{h}}{\varepsilon_{h}+\left(\varepsilon_{N P}-\varepsilon_{h}\right) P_{j}}
$$

with the dielectric function of nanoparticles given by Eq. (S.4). After simple algebraic manipulations, it is possible to calculate the values of:

$$
\begin{gathered}
\left.\frac{\partial^{2} \sigma_{j}\left(\varepsilon_{N P}\right)}{\partial x^{2}}\right|_{x=0}=\frac{3}{2} \frac{\left[\beta_{j}\left(\varepsilon_{N P}\right)\right]^{2}}{\varepsilon_{h}} \chi_{N P}^{(3)}, \\
\left.\frac{\partial^{4} \sigma_{j}\left(\varepsilon_{N P}\right)}{\partial x^{4}}\right|_{x=0}=\frac{5 !}{8} \frac{\left[\beta_{j}\left(\varepsilon_{N P}\right)\right]^{2}}{\varepsilon_{h}}\left[\chi_{N P}^{(5)}-\frac{9}{10} P_{j} \frac{\beta_{j}\left(\varepsilon_{N P}\right)}{\varepsilon_{h}}\left(\chi_{N P}^{(3)}\right)^{2}\right], \\
\left.\frac{\partial^{2}\left(\left|\beta_{j}\left(\varepsilon_{N P}\right)\right|^{2}\right)}{\partial x^{2}}\right|_{x=0}=-3\left|\beta_{j}\left(\varepsilon_{N P}\right)\right|^{2} \operatorname{Re}\left[P_{j} \frac{\beta_{j}\left(\varepsilon_{N P}\right)}{\varepsilon_{h}} \chi_{N P}^{(3)}\right] .
\end{gathered}
$$

Finally, by substituting Eqs. (S.12)-(S.14) in Eqs. (S.9) and (S.10), we obtain the effective third- and fifth-order susceptibilities for colloids containing randomly distributed nanoellipsoids suspended in a linear host medium, written as: 


$$
\begin{gathered}
\chi_{\text {eff }}^{(3)}=f \sum_{j, k=1}^{3}\left[\frac{\left[\beta_{j}\left(\varepsilon_{N P}\right)\right]^{2}}{3} \frac{\left|\beta_{k}\left(\varepsilon_{N P}\right)\right|^{2}}{3}\right] \chi_{N P}^{(3)}, \\
\chi_{\text {eff }}^{(5)}=f \sum_{j=1}^{3}\left[\left[\chi_{N P}^{(5)}-\frac{9}{10} P_{j} \frac{\beta_{j}\left(\varepsilon_{N P}\right)}{\varepsilon_{h}}\left(\chi_{N P}^{(3)}\right)^{2}\right] \frac{\left[\beta_{j}\left(\varepsilon_{N P}\right)\right]^{2}}{3}\left(\sum_{k=1}^{3} \frac{\left|\beta_{k}\left(\varepsilon_{N P}\right)\right|^{2}}{3}\right)^{2}\right] \\
-\frac{9}{10} \chi_{\text {eff }}^{(3)} \sum_{j=1}^{3}\left[\frac{\left|\beta_{j}\left(\varepsilon_{N P}\right)\right|^{2}}{3} 2 \operatorname{Re}\left(P_{j} \frac{\beta_{j}\left(\varepsilon_{N P}\right)}{\varepsilon_{h}} \chi_{N P}^{(3)}\right)\right] .
\end{gathered}
$$

\section{The critical point model and the Drude free-electron model}

The frequency dependence of the nanoparticles dielectric function used in the manuscript was represented by

$$
\varepsilon_{N P}(\omega)=\varepsilon_{b u l k}(\omega)+i \frac{A \omega_{p}^{2} v_{F}}{\omega^{3} R_{N P}},
$$

where the $\varepsilon_{\text {bulk }}$, for the specific case of gold, can be simulated by using the critical point model in conjunction with the Drude free-electron model, given by:

$$
\varepsilon_{\text {bulk }}(\omega)=\varepsilon_{\text {Drude }}(\omega)+\sum_{j=1}^{2} G_{j}(\omega),
$$

with

$$
\varepsilon_{\text {Drude }}(\omega)=\varepsilon_{\infty}-\frac{\omega_{p}^{2}}{\omega^{2}+i \Gamma_{0} \omega},
$$

and

$$
G_{j}(\omega)=C_{j}\left[e^{i \phi_{j}}\left(\omega_{j}-\omega-i \Gamma_{j}\right)^{\mu_{j}}+e^{-i \phi_{j}}\left(\omega_{j}+\omega+i \Gamma_{j}\right)^{\mu_{j}}\right] .
$$

The parameters used to fit the theoretical model with the experimental data reported by Johnson and Christy (Ref. [64]) are given in Table S1, in accordance with those reported in Ref. [64]. Figure S4 shows a comparison between the theoretical results and the experimental measurements. 
Table S1. Parameters used in the critical point model in conjunction with the Drude free-electron model

\begin{tabular}{|c|c|c|c|c|}
\hline Model & Parameters & Symbols & \multicolumn{2}{|c|}{ Values } \\
\hline \multirow{3}{*}{$\begin{array}{l}\text { Drude free- } \\
\text { electron }\end{array}$} & $\begin{array}{l}\text { High-frequency limit } \\
\text { dielectric constant }\end{array}$ & $\varepsilon_{\infty}$ & \multicolumn{2}{|c|}{1.54} \\
\hline & Plasma frequency [Hz] & $\omega_{P}$ & \multicolumn{2}{|c|}{$1.32 \times 10^{16}$} \\
\hline & Damping term $[\mathrm{Hz}]$ & $\Gamma$ & \multicolumn{2}{|c|}{$1.30 \times 10^{14}$} \\
\hline \multirow{6}{*}{ Critical point } & & & $j=1$ & $j=2$ \\
\hline & Phase & $\phi_{j}$ & $-\pi / 4$ & $-\pi / 4$ \\
\hline & Order of the pole & $\mu_{j}$ & -1 & -1 \\
\hline & $\begin{array}{l}\text { Interband transition } \\
\text { frequency }[\mathrm{Hz}]\end{array}$ & $\omega_{j}$ & $4.01 \times 10^{15}$ & $5.80 \times 10^{15}$ \\
\hline & $\begin{array}{c}\text { Transition broadenings } \\
{[\mathrm{Hz}]}\end{array}$ & $\Gamma_{j}$ & $9.92 \times 10^{14}$ & $1.78 \times 10^{15}$ \\
\hline & Amplitude $[\mathrm{Hz}]$ & $\overline{C_{j}}$ & $5.09 \times 10^{15}$ & $6.38 \times 10^{15}$ \\
\hline
\end{tabular}
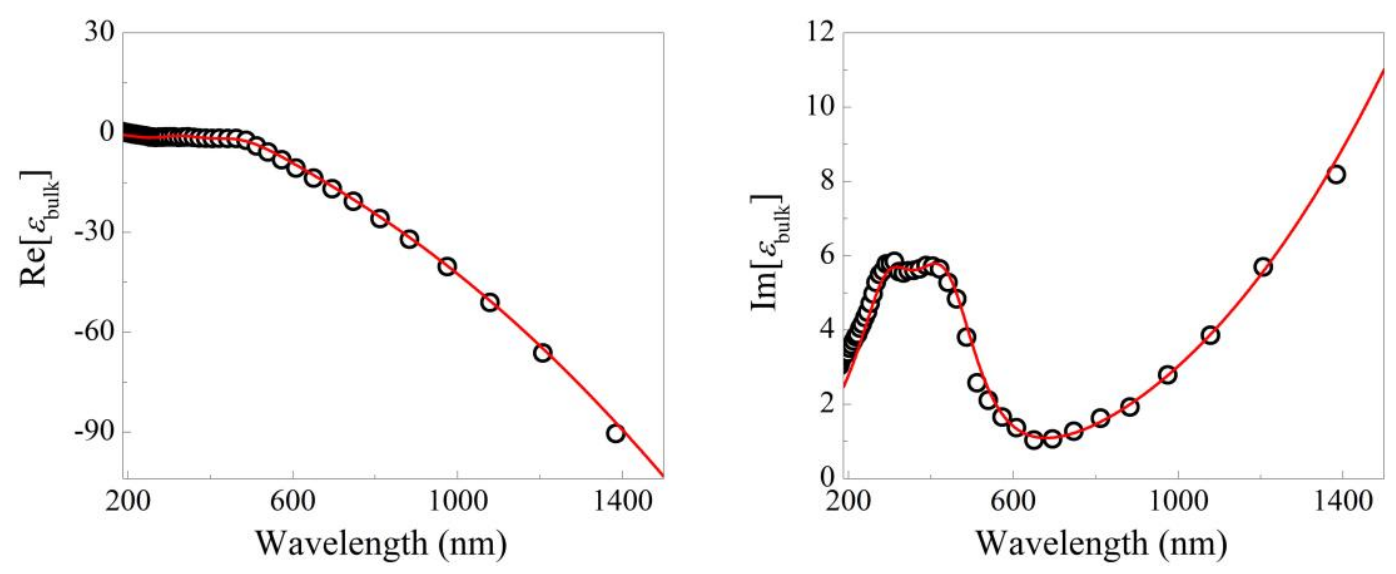

FIG. S4: Comparison between the experimental data (black circles) reported by Johnson and Christy and the theoretical results using the Eq. (S.18), for the real and imaginary parts of the bulk dielectric function of gold. 